\title{
Asymptotic Normality of Finite Fourier Transforms of Stationary Generalized Processes*
}

\author{
DAVID R. BRILLINGER \\ The University of California, Berkeley \\ Communicated by $M$. Rosenblatt
}

\begin{abstract}
This paper indicates a mixing condition under which a net of Fourjer transforms, of a stationary generalized process over an abelian locally compact group, has a limiting normal distribution.
\end{abstract}

\section{INTRODUCTION}

Finite Fourier transforms of stationary mixing processes have been shown to be asymptotically normal in quite a variety of circumstances. The case of a time series $X(t)$ with $t$ in $R$ is considered in, for example, Leonov and Shiryaev [12], Picinbono [16], Rosenblatt [19], Rozanov [21]. The case of $t$ in $Z$ is considered in Hannan [8, Chap.IV], in Hannan and Thomson [9], in Brillinger [5]. The case of $t$ in $R^{p}$ is investigated in Brillinger [2] and in Pichard [17]. Morettin [13] considers the case of $t$ in a non-compact locally compact second countable group. In extensions of another sort Brillinger [3] indicates conditions leading to asymptotic normality in the case of stationary random measures on $R$ and Brillinger [4] in the case of stationary random Schwartz distributions with $t$ in $R^{p}$. This paper provides a central limit theorem for Fourier transforms of stationary random Schwartz-Bruhat distributions over a locally compact abelian group $G$. In the general case nets of Fourier transforms are shown to be asymptotically normal. When $G$ is $\sigma$-compact the nets become sequences. The Fourier transforms need not be asymptotically normal. Rosenblatt $[20]$ derives a non-Gaussian limit for the transform of a process with long range dependence.

* Prepared with the partial support of National Science Foundation Grant PFR 7901642.

Received April 1981; revised May 24, 1981.

AMS 1970 subject classification: Primary 60B15, 60G20, 60F05; Secondary 43A25, $60 \mathrm{M} 15$.

Key words and phrases: asymptotic normality, central limit theorem, Fourier transform. generalized process, group, mixing. 
In this work $G$ denotes a locally compact abelian group, $\boldsymbol{A}$ denotes its dual. Haar measures on these two groups are denoted by $d g$ and $d \lambda$, respectively. The group operation is denoted by " + ". Characters of the groups are denoted by $\langle g, \lambda\rangle$ for $g$ in $G$ and $\lambda$ in $\Lambda$. The Fourier transform of a function $\psi$ in $L_{1}(G)$ is defined by

$$
\Psi(\lambda)=\int\langle g,-\lambda\rangle \psi(g) d g
$$

If $\Psi$ belongs to $L_{1}(\Lambda)$ one has the inversion formula

$$
\psi(g)=\int\langle g, \lambda\rangle \Psi(\lambda) d \lambda
$$

In the case of Euclidean space, the infinitely differentiable functions of rapid decrease provide a setting "par excellence" (Schwartz [22, p. 104]) for harmonic analysis. Similar functions exist in the group case and may be defined as follows (Osborne [15]).

First one defines $A(G)$ the space of functions whose $L_{\infty}$ norm decreases rapidly off powers of a compact set. Specifically $\psi \in A(G)$ if there exists a compact set $\bar{C}(\psi) \subset \bar{G}$ such that for each positive integer $n$ there is a constant $M_{n}$ such that for each integer $k \geqslant 1$

$$
\left\|\left.\psi\right|_{G-C(\phi)}\right\|_{\infty} \leqslant M_{n} k^{-n}
$$

One has, for example, $A(G) \subset L_{p}(G), p \geqslant 1, L_{\infty}(G) \cdot A(G) \subset A(G)$ and $A(G) * A(G) \subset A(G) . A(G)$ is translation invariant.

The Schwartz-Bruhat space, $S(G)$, of rapidly decreasing test functions consists of functions $\psi \in A(G)$ with Fourier transforms $\Psi \in A(\Lambda)$. The space has a topology arising from the inequalities defining $A(G)$ and $A(\Lambda)$. One has $S(G) \subset A(G), A(G) * S(G) \subset S(G), S(G) \cdot S(G) \subset S(G)$. $S(G)$ is translation invariant.

A Schwartz-Bruhat tempered distribution is a continuous linear functional on $S(G)$. Such a distribution $x(\psi), \psi \in S(G)$ has a Fourier transform $X(\Psi)$, $\Psi \in S(A)$, whose (inverse) Fourier transform is $x(\psi)$ in turn.

A final space that will be made use of is $D(G)=S(G) \cap K(G)$, where $K(G)$ denotes the space of continuous functions of compact support on $G$. One introduces a finer topology on $D(G)$ than that of $S(G)$. (See the references below.) One has $D(G) * D(G) \subset D(G)$ and $D(G) \cdot D(G) \subset D(G)$, and $D(G)$ is dense in $S(G)$. A Schwartz-Bruhat (ordinary) distribution is a continuous linear functional on $D(G)$. A tempered distribution is an (ordinary) distribution which is continuous with respect to the $S(G)$ topology. 
The above concepts are discussed in Bruhat [6], Wawrzynczyk [25], Osborne [15], Argabright and Gil de Lampard [1].

\section{Stationary Generalized Processes}

This paper is concerned with random Schwartz-Bruhat distributions. These are defined as mean-square continuous (in the $D(G)$-topology) random linear functionals on $D(G)$, whose values are equivalence classes of complex-valued random variables of second-order defined on some probability space $(\Omega, \mathbb{Q}, P)$ and which form a Hilbert space $L_{2}(\Omega)$. (See Ponomarenko [18].) In the stationary case the covariance function of such a process has a spectral representation

$$
\operatorname{cov}\left\{x\left(\psi_{1}\right), x\left(\psi_{2}\right)\right\}=\int \Psi_{1}(\lambda) \overline{\Psi_{2}(\lambda)} F_{2}(d \lambda) .
$$

$F_{2}$ has a non-negative measure on $A$ and the process itself has a spectral representation

$$
x(\psi)=\int \Psi(\lambda) Z(d \lambda)=X(\Psi)
$$

with $Z$ a stochastic measure satisfying $\operatorname{cov}\{Z(A), Z(B)\}=F_{2}(A \cap B)$. The domain of $x$ may be expanded to $\psi \in S(G)$ and to $\psi$ with $\Psi \in L_{2}\left(F_{2}\right)$. In the case that the process $x$ is real $Z(d \lambda)=\overline{Z(-d \bar{\lambda})}$. (See Ponomarenko [18].)

The above spectral representations were developed by Kampe de Feriet [11] for the case of a stationary ordinary process over a group $G$ and by Niemi $[14]$ for the case of a stationary random measure on a group $G$ and by Cramér [7] originally.

A mixing condition will be required in order to develop the central limit theorems of this paper. It is,

AsSUMPTION I. $x$ is a real stationary generalized process. For $k=1,2, \ldots$ the cumulant of order $k+1$ of $x(\psi)$ is assumed to exist and be given by

$$
\int \cdots \int \Psi\left(\lambda_{1}\right) \cdots \Psi\left(\lambda_{k}\right) \overline{\Psi\left(\lambda_{1}+\cdots+\lambda_{k}\right)} f_{k+1}\left(\lambda_{1}, \ldots, \lambda_{k}\right) d \lambda_{1} \cdots d \lambda_{k}
$$

with

$$
\text { vrai sup }\left|\Psi\left(\lambda_{1}\right) \cdots \Psi\left(\lambda_{k}\right) \Psi\left(\lambda_{1}+\cdots+\lambda_{k}\right) f_{k+1}\left(\lambda_{1}, \ldots, \lambda_{k}\right)\right|<\infty
$$

for all $\Psi \in S(\Lambda)$. 
The functions $\tilde{f}_{k+1}$ are called the cumulant spectra of the process $x$. They are given in terms of the stochastic measure $Z$ by

$$
\begin{aligned}
\operatorname{cum} & \left\{\left(d \lambda_{1}\right), \ldots, Z\left(d \lambda_{k+1}\right)\right\} \\
& =\delta\left(\lambda_{1}+\cdots+\lambda_{k+1}\right) f_{k+1}\left(\lambda_{1}, \ldots, \lambda_{k}\right) d \lambda_{1} \cdots d \lambda_{k+1},
\end{aligned}
$$

where $\delta$ is the Dirac delta function.

Making an observation on the process $x$ will be viewed as being able to compute $x(\psi)$ for all $\psi$ in $D(G)$ having support within a given compact set $T$, (the domain of observation). The sample sum will be viewed as the value $x(\psi)$ with $\psi$ near 1 throughout $T$. The finite Fourier transform will be taken to be

$$
x(\langle g,-\omega\rangle \psi)=\int \Psi(\omega-\lambda) Z(d \lambda)=X(\Psi(\omega-\cdot))
$$

for such a $\psi$. The function $\psi$ will need to be in $D(G)$ and will be called a taper.

In the case that $G$ is not compact and that $T$ is large the finite Fourier transform (2.5) might be expected to be approximately normally distributed when the process $x$ is mixing. The notion that $T$ is large will be formalized by basing the Fourier transform on a net of tapers $\left\{\psi_{a}\right\}$ such that $\lim \psi_{a}(g)=1$ (pointwise) for all $g \in G$. (In the case that $G$ is $\sigma$-compact the net may be taken to be a sequence.) It will be seen that nets of tapers exist such that the finite Fourier transform is asymptotically normal.

\section{The Asymptotic Normality}

The following regularity conditions will be required in connection with the net of tapers.

Assumption II. $\left\{\Psi_{\alpha}\right\}$ is a net of functions in $S(\Lambda)$ with the property that $\Psi_{\alpha}$ may be written $\Psi_{\alpha}=\Phi_{\alpha} \Psi$ with $\Phi_{\alpha}, \Psi \in S(\Lambda), \Psi(0)=1$

$$
\int\left|\Phi_{\alpha}\right|^{2}=1, \quad \lim \int_{\Lambda / U}\left|\Phi_{\alpha}\right|^{2}=0
$$

for $U$ any neighborhood of 0 in $A$ and

$$
\lim || \Phi_{\alpha} \mid=0
$$


In Appendix 1 of the paper such a net will be constructed in the case of non-compact $G$ with the further properties that $\psi_{\alpha} \in D(G)$ and that $\psi_{\alpha}(g)$ is proportional to a function with limit $=1$ for $g \in G$. In the case of $\sigma$-compact $G$, the net may be taken to be a sequence.

The principal result of the paper may now be stated.

THEOREM. Let the stationary generalized process $x$ satisfy Assumption I and have mean 0. Suppose further that the second-order spectrum $f_{2}(\lambda)$ is

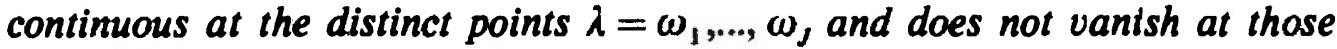
points. Let the net (or sequence) $\left\{\Psi_{\alpha}\right\}$ satisfy Assumption II. Then the finite Fourier transforms $X\left(\Psi_{\alpha}\left(\omega_{j}-\cdot\right)\right), j=1, \ldots, J$ are asymptotically independent normals with zero means and variances $f_{2}\left(\omega_{j}\right), j=1, \ldots, J$, respectively.

Proof. The argument proceeds by showing that the cumulants of order greater than 2 tend to 0 and that those of order 2 tend to the values indicated. Because the normal distribution is determined by its cumulants (those of order greater than 2 vanish), and because the cumulants exist and tend to 0 as indicated, the corresponding net of probability measures is tight and asymptotic normality follows. The argument is carried through for the univariate case. The joint normality argument may be reduced to this case. Using abbreviated notation, one has for cumulants of order $k+1$

$\left|\operatorname{cum}\left\{X\left(\Psi_{\alpha}\right), \ldots, X\left(\Psi_{\alpha}\right)\right\}\right|$

$$
=\left|\int \Psi_{\alpha}\left(\lambda_{1}\right) \cdots \Psi_{\alpha}\left(\lambda_{k}\right) \overline{\Psi_{\alpha}\left(\lambda_{1}+\cdots+\lambda_{k}\right)} f_{k+1}\left(\lambda_{1}, \ldots, \lambda_{k}\right) d \lambda_{1} \cdots d \lambda_{k}\right|
$$

from (2.3)

$$
\begin{aligned}
& \leqslant M \int\left|\Phi_{\alpha}\left(\lambda_{1}\right) \cdots \Phi_{\alpha}\left(\lambda_{k}\right) \Phi_{\alpha}\left(\lambda_{1}+\cdots+\lambda_{k}\right)\right| d \lambda_{1} \cdots d \lambda_{k} \text { from }(2.4) \\
& \leqslant M\left(\int\left|\Phi_{\alpha}(\lambda)\right|^{(k+1) / k} d \lambda\right)^{k} \text { from Appendix } 2 .
\end{aligned}
$$

Now $\left(\int|\Phi|^{(k+1) / k}\right)^{k} \leqslant \int|\Phi|^{2}\left(\int|\Phi|\right)^{k-1}$ by Holder's Inequality and so the cumulant indicated tends to 0 from (3.2) in the case $k>1$. (Use is also made here of the fact that the space $S(G)$ is translation invariant.)

In the case $k=1$

$$
\begin{aligned}
\operatorname{var} X\left(\Psi_{\alpha}(\omega-\cdot)\right) & =\int\left|\Psi_{\alpha}(\omega-\lambda)\right|^{2} f_{2}(\lambda) d \lambda \\
& =\int\left|\Phi_{\alpha}(\omega-\lambda)\right|^{2}|\Psi(\omega-\lambda)|^{2} f_{2}(\lambda) d \lambda
\end{aligned}
$$


tending to $f_{2}(\omega)$ at points of continuity of $f_{2}$ in view of (3.1), the approximate identity nature of the net $\left|\Phi_{\alpha}\right|^{2}$. In the covariance case one has

$$
\begin{aligned}
& \left|\operatorname{cov}\left\{X\left(\Psi_{\alpha}\left(\omega_{1}-\cdot\right)\right), X\left(\Psi_{\alpha}\left(\omega_{2}-\cdot\right)\right)\right\}\right| \\
& \quad=\left|\int \Psi_{\alpha}\left(\omega_{1}-\lambda\right) \overline{\Psi_{\alpha}\left(\omega_{2}-\lambda\right)} f_{2}(\lambda) d \lambda\right| \\
& \quad \leqslant M \int\left|\Phi_{\alpha}\left(\omega_{1}-\lambda\right) \Phi_{\alpha}\left(\omega_{2}-\lambda\right)\right| d \lambda
\end{aligned}
$$

from (2.4). This last integral may be seen to tend to 0 for $\omega_{1} \neq \omega_{2}$ by splitting it up into an integral over a neighborhood of $\omega_{1}$ not containing $\omega_{2}$ and a remainder and then using Schwarz's Inequality separately on each integral.

This completes the proof of the Theorem.

\section{EXTENSIONS}

The theorem extends to the case of vector-valued processes by means of the same arguments. This extension includes the complex-valued case.

\section{APPENDIX 1}

This Appendix demonstrates the existence of a net of functions, $\Psi_{\alpha} \in S(\Lambda)$ satisfying Assumption II.

Simon [24] and Hewitt and Ross [10, p. 298] construct nets, $\Gamma_{\alpha} \in L_{1}(\Lambda)$, such that: (i) $\Gamma_{\alpha} \geqslant 0, \int \Gamma_{\alpha}(\lambda) d \lambda=1$; (ii) $0 \leqslant \gamma_{\alpha}(g) \leqslant 1, \gamma_{\alpha} \in K(G)$; (iii) given $U$ a neighborhood of 0 in $A$ and $\beta>0$, there is an $\alpha_{0}$ such that for $\alpha>\alpha_{0}$ one has $\int_{U} \Gamma_{\alpha}(\lambda) d \lambda>1-\beta$ and $\Gamma_{\alpha}(\lambda)<\beta$ for $\lambda \in \Lambda / U$; (iv) $\lim \gamma_{\alpha}(g)=1$ uniformly on compact subsets of $G$; and $(v)$ the net becomes a sequence for $\boldsymbol{G} \boldsymbol{\sigma}$-compact.

Bruhat [6] demonstrates the existence of functions $\theta \in D(G)$ such that $\theta \geqslant 0$ and $\int \theta(g) d g=1$ and one has $|\theta| \leqslant 1$.

Now set

$$
\phi_{\alpha}=\gamma_{\alpha} * \theta /\left(\int\left(\gamma_{\alpha} * \theta\right)^{2}\right)^{1 / 2}, \quad \phi_{\alpha}=\Gamma_{\alpha} \theta /\left(\int\left(\Gamma_{\alpha} \theta\right)^{2}\right)^{1 / 2} .
$$

One sees that $\gamma_{\alpha} * \theta(g) \geqslant 0$ and tends to $\int \theta(g) d g=1$. This implies that $\int \Gamma_{\alpha}^{2} \theta^{2}$ tends to $\infty$ for non-compact $G$. Further, from (iii), 


$$
\int_{\Lambda / U} \Gamma_{\alpha}^{2} \Theta^{2} \leqslant \beta(1-\beta)
$$

giving (3.1). Next, $\int\left|\Gamma_{\alpha} \Theta\right| \leqslant \int \Gamma_{\alpha} \leqslant 1$ and (3.2) follows.

The construction is completed by taking $\Psi=\Theta$.

\section{APPENDIX 2}

LemMA. Let $k$ be a positive integer and $\Phi$ in $L_{p}(\Lambda)$ with $p=(k+1) / k$. Then

$$
\int \cdots \int\left|\Phi\left(\lambda_{1}\right) \cdots \Phi\left(\lambda_{k}\right) \Phi\left(\lambda_{1}+\cdots+\lambda_{k}\right)\right| d \lambda_{1} \cdots d \lambda_{k} \leqslant\left(\int|\Phi(\lambda)|^{p} d \lambda\right)^{k} .
$$

Proof. The result follows from Holder's Inequality, namely,

(a) $\|f g\|_{1} \leqslant\|f\|_{p}\|g\|_{q}, 1 / p+1 / q=1$, Young's inequality (Segal and Kunze [23, p. 204], namely,

(b) $\|f * g\|_{r} \leqslant\|f\|_{p}\|g\|_{q}, 1 / r=1 / p+1 / q-1 \geqslant 0$ and Fubini. Define $H_{0}(\lambda)=\Phi(\lambda)$,

$$
H_{j+1}(\lambda)=\int\left|\Phi(\lambda+\mu) H_{j}(\mu)\right| d \mu
$$

and note that if the integral exists it is given by $\int\left|\Phi(\lambda) H_{k-1}(\lambda)\right| d \lambda$. Now,

$$
\begin{aligned}
\int\left|\Phi(\lambda) H_{k-1}(\lambda)\right| d \lambda & \leqslant\|\Phi\|_{p}\left\|H_{k-1}\right\|_{k+1} \\
\left\|H_{k-1}\right\|_{k+1} & \leqslant\|\Phi\|_{p}\left\|H_{k-2}\right\|_{(k+1) / 2} \leqslant \cdots \leqslant\|\Phi\|_{p}^{k-1}\left\|H_{0}\right\|_{(k+1) / k} \\
& =\|\Phi\|_{p}^{k} .
\end{aligned}
$$

\section{REFERENCES}

[1] ARgABRIGHT, L., AND GIL DE LAMADRID, J. (1974). Fourier analysis of unbounded measures on locally compact abelian groups. Mem. Amer. Math. Soc. 145.

[2] BRILLINGER, D. R. (1970). The frequency analysis of relations between stationary spatial series. In Proceedings, Twelfth Biennial Seminar Canadian Mathematics Congress (R. Pyke, Ed. ) pp. 39-81. Canad. Math. Congress, Montreal.

131 BRILLunger, D. R. (1972). The spectral analysis of stationary interval functions. In Proceedings, Sixth Berkeley Symposium Math. Statist. Prob. (L. M. Le Cam, J. Neyman, and E. L. Scott, Eds.), pp. 483-513. Univ. Calif. Press, Berkeley.

14) Brilunger, D. R. (1974). The Fourier analysis of stationary processes. Proc. Inst. Electr. Engrs. 62, 1628-1643. 
[5] Brillinger, D. R. (1975). Time Sertes: Data Analysis and Theory. Holt, Rinehart \& Winston, New York.

[6] Bruhat, F. (1961). Distributions sur un groupe localement compact et applications à l'étude des représentations des groupes p-adiques. Bull. Soc. Math. France 89, 43-75.

17] Cramér, H. (1942). On harmonic analysis in certain functional spaces. Arkiv Math. Astron. Fys. 28, 1-7.

[8] Hannan, E. J. (1970). Multiple Time Series. Wiley, New York.

[9] Hannan, E. J., and Thomson, P. J. (1971). Spectral inference over narrow bands. $J$. Appl. Probab. 8, 157-169.

[10] Hewitt, E., AND Ross, K. A. (1970). Abstract Harmonic Analysis, II. Springer-Verlag, Berlin.

111 Kampé de Fériet, J. (1948). Analyse harmonique des fonctions aleatoires stationaires d'ordre 2 sur un group abélien compact. C.R. Acad. Sci. Paris 226, 868-870.

112] Leonov, V. P. AND Shiryaev, A. N. (1960). Some problems in the spectral theory of higher-order moments, II. Theory Probab. Appl. 4, 319-329.

113) Morettin, P. A. (1980). Homogeneous random processes on locally compact abelian groups. Anais Acad. Brasil. Ciênc. 52, 1-6.

[14] Niemi, H. (1978). Stationary vector measures and positive definite translation invariant bimeasures. Ann. Acad. Sci. Fenn. Ser. A 4, 209-226.

[15] Osborne, M. S. (1975). On the Schwartz-Bruhat space and the Paley-Wiener theorem for locally compact abelian groups. J. Funct. Anal. 19, 40-49.

[16] Picinbono, B. (1960). Tendance vers le caractère gaussien par filtrage sélectif. C.R. Acad. Sci. Paris 250, 1174-1176.

[17] Pichard, J.-F. (1974). Normalisation par filtrage selectif de fonctions aléatoires de plusieurs variables réeles de classe $\Delta^{(\infty)}$. C.R. Acad. Sci. Paris 278, 443-446.

[18] Ponomarenko, A. I. (1974). Harmonic analysis of generalized wide-sense homogeneous random fields on a locally compact commutative group. Theoret. Prob. Math. Statist. 4, 119-137.

[19] Rosenblatt, M. (1961). Some comments on narrow band-pass filters. Quart. Appl. Math. 18, 387-394.

[20] Rosenblatt, M. (1981). Limit theorems for Fourier transforms of functionals of Gaussian sequences. Z. Wahrsch. Verw. Gebiete 55, 123-132.

121] Rozanov, Yu. A. (1961). An application of the central limit theorem. In Proceedings, Fourth Berkeley Symposium Math. Statist. Prob. (J. Neyman, Ed.) Vol. 1, pp. 445-454.

[22] Schwartz, L. (1959). Théorie des distributions, II. Hermann, Paris.

[23] Segal, I. E., AND Kunze, R. A. (1978). Integrals and Operators. McGraw-Hill, New York.

[24] Simon, A. B. (1966). Cesàro summability of groups: characterization and inversion of Fourier transforms. In Function Algebras, pp. 208-215. Scott, Foresman, New York.

[25] WaWrzyNCzYX, A. (1968). On tempered distributions and Bochner-Schwartz theorem on arbitrary locally compact abelian groups. Colloq. Math. 19, 305-318. 